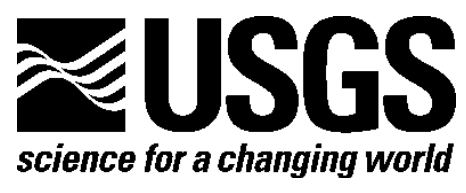

\title{
Petrologic and Isotopic Data from the Cretaceous (Campanian) Blackhawk Formation and Star Point Sandstone (Mesaverde Group), Wasatch Plateau, Utah
}

By Neil S. Fishman, Christine E. Turner, and Fred Peterson

Open-File Report 2013-1254

U.S. Department of the Interior

U.S. Geological Survey 


\section{U.S. Department of the Interior \\ SALLY JEWELL, Secretary}

\section{U.S. Geological Survey \\ Suzette M. Kimball, Acting Director}

U.S. Geological Survey, Reston, Virginia: 2013

For more information on the USGS-the Federal source for science about the Earth, its natural and living resources, natural hazards, and the environment-visit http://www.usgs.gov or call 1-888-ASK-USGS

For an overview of USGS information products, including maps, imagery, and publications, visit $h$ ttp://www.usgs.gov/pubprod

To order this and other USGS information products, visit http://store.usgs.gov

Suggested citation:

Fishman, N.S., Turner, C.E., and Peterson, Fred, 2013, Petrologic and isotopic data from the Cretaceous (Campanian) Blackhawk Formation and Star Point Sandstone (Mesaverde Group), Wasatch Plateau, Utah: U.S. Geological Survey Open-File Report 2013-1254, 15 p., 1 plate, http://dx.doi.org/ofr20131254.

Any use of trade, product, or firm names is for descriptive purposes only and does not imply endorsement by the U.S. Government.

Although this report is in the public domain, permission must be secured from the individual copyright owners to reproduce any copyrighted material contained within this report. 


\section{Contents}

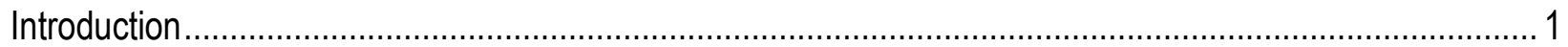

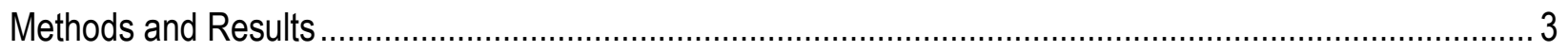

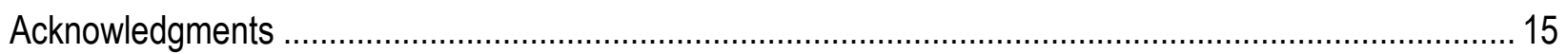

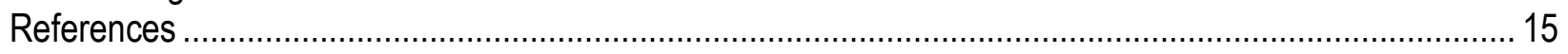

\section{Figures}

Figure 1. Map showing outcrop and drill core locations across the study area from which samples of the Cretaceous Blackhawk Formation and Star Point Sandstone were obtained.....

Figure 2. Generalized stratigraphic column showing the relative positions and ages of the units from which samples were taken for this study.

Figure 3. Summary of the paragenetic sequence of alterations in sandstone beds in the Blackhawk Formation and Star Point Sandstone

\section{Tables}

Table 1. Location information for outcrops from which samples of the Blackhawk Formation and (or) Star Point Sandstone were collected

Table 2. Location information for core samples of the Cretaceous Blackhawk Formation that were used in this study

Table 3. Point count data for sandstones from either the Blackhawk Formation or Star Point Sandstone .. 9

Table 4. Blackhawk Formation samples from which material was obtained from apertures and analyzed by X-ray diffraction and (or) scanning electron microscope

Table 5. Carbon and oxygen isotopic composition of calcite from either cleats or fractures in the Blackhawk Formation or Star Point Sandstone

\section{Plate}

Measured sections of the Blackhawk Formation and Star Point Sandstone from sampled localities across the Wasatch Plateau link 


\title{
Petrologic and Isotopic Data from the Cretaceous (Campanian) Blackhawk Formation and Star Point Sandstone (Mesaverde Group), Wasatch Plateau, Utah
}

\author{
By Neil S. Fishman, Christine E. Turner, and Fred Peterson
}

\section{Introduction}

The presence of discrete minerals associated with coal—whether (1) detrital or authigenic constituents of the coals or in thin mudstone or siltstone units interbedded with coals, or (2) authigenic phases that formed along cleats - might influence its utilization as an energy resource. The build-up of sintered ash deposits on the surfaces of heat exchangers in coal-fired power plants, due to the alteration of minerals during combustion of the coal (Reid, 1981; Finkelman and Dulong, 1989), can seriously affect the functioning of the boiler and enhance corrosion of combustion equipment (Honea and others, 1982; Vaninetti and Busch, 1982). In particular, the presence of sodium in coals has been considered a key factor in the fouling of boilers (Ely and Barnhart, 1963); however, other elements (such as calcium or magnesium) and the amount of discrete minerals burned with coal can also play a significant role in the inefficiency of and damage to boilers (Finkelman and Dulong, 1989).

Previous studies of the quality of coals in the Cretaceous (Campanian) Blackhawk Formation of the Wasatch Plateau, Utah, revealed that the sodium content of the coals varied across the region (Finkelman, 1988, 1991). To better understand the origin and distribution of sodium in these coals, petrologic studies were undertaken within a sedimentological framework to evaluate the timing and geochemical constraints on the emplacement of sodium-bearing minerals, particularly analcime, which previously had been identified in coals in the Blackhawk Formation (Finkelman, 1988, 1991). Further, the study was broadened to include not just coals in the Blackhawk Formation from various localities across the Wasatch Plateau (fig. 1), but also sandstones interbedded with the coals as well as sandstones in the underlying Star Point Sandstone (fig. 2). The alteration history of the sandstones in both formations was considered a key component of this study because it records the nature and timing of fluids passing through them and the associated precipitation of sodium-bearing minerals; thus, the alteration history could place constraints on the distribution and timing of sodium mineralization in the interbedded or overlying Blackhawk coals. Although some preliminary results were previously presented at scientific meetings (Turner and others, 1997; Fishman and others, 1999), the petrologic and geochemical data have not been fully compiled and reported. The purpose of this report is to present the methods of data acquisition and the results of petrologic and isotopic analyses on coal and sandstone samples from the Blackhawk Formation as well as sandstones of the underlying Star Point Sandstone. 

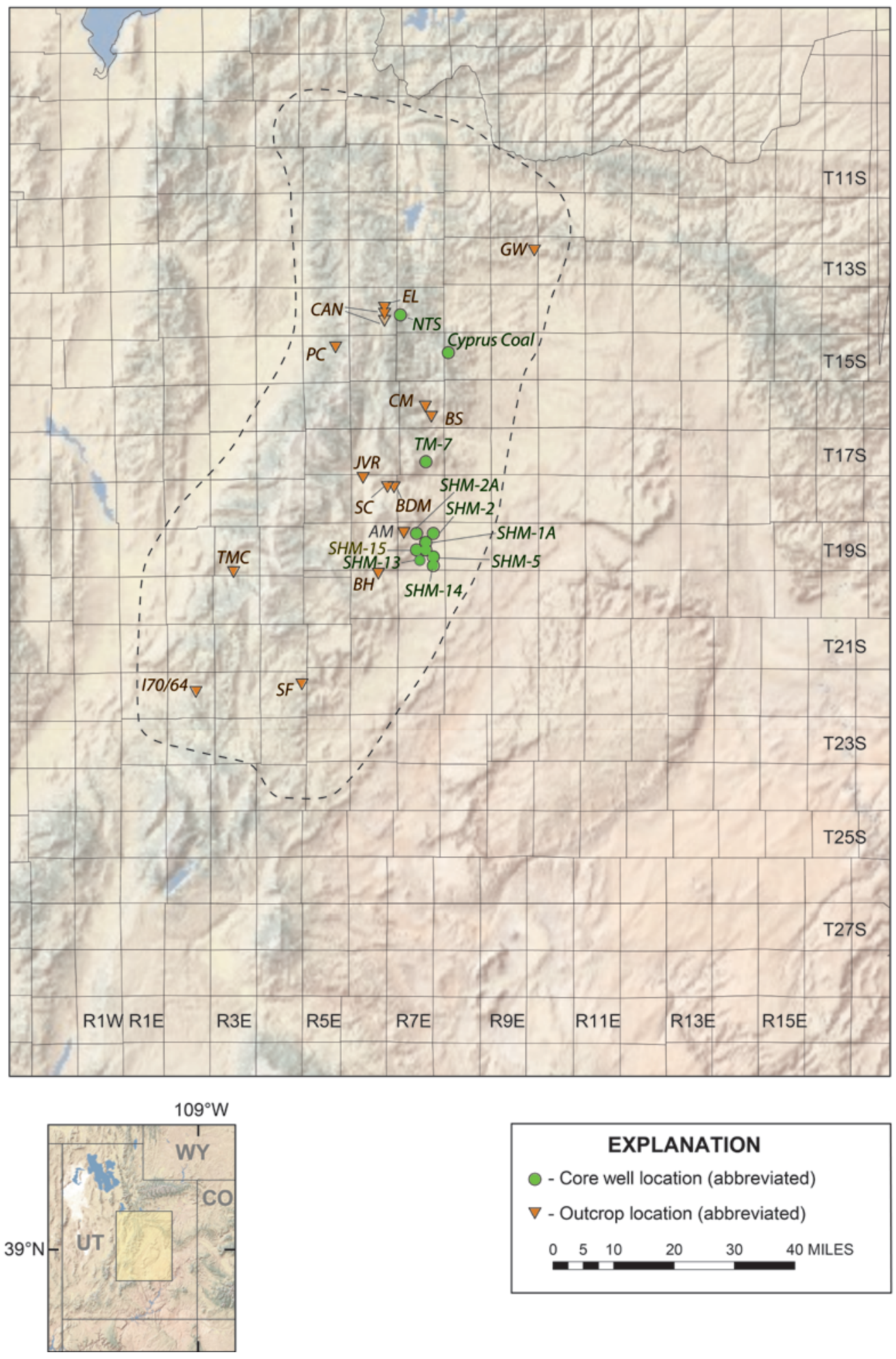

Figure 1. Map showing outcrop and drill core locations across the study area from which samples of the Cretaceous Blackhawk Formation and Star Point Sandstone were obtained. See tables 1 and 2 for location abbreviations. Generalized outline of the Wasatch Plateau also shown (dashed line). 


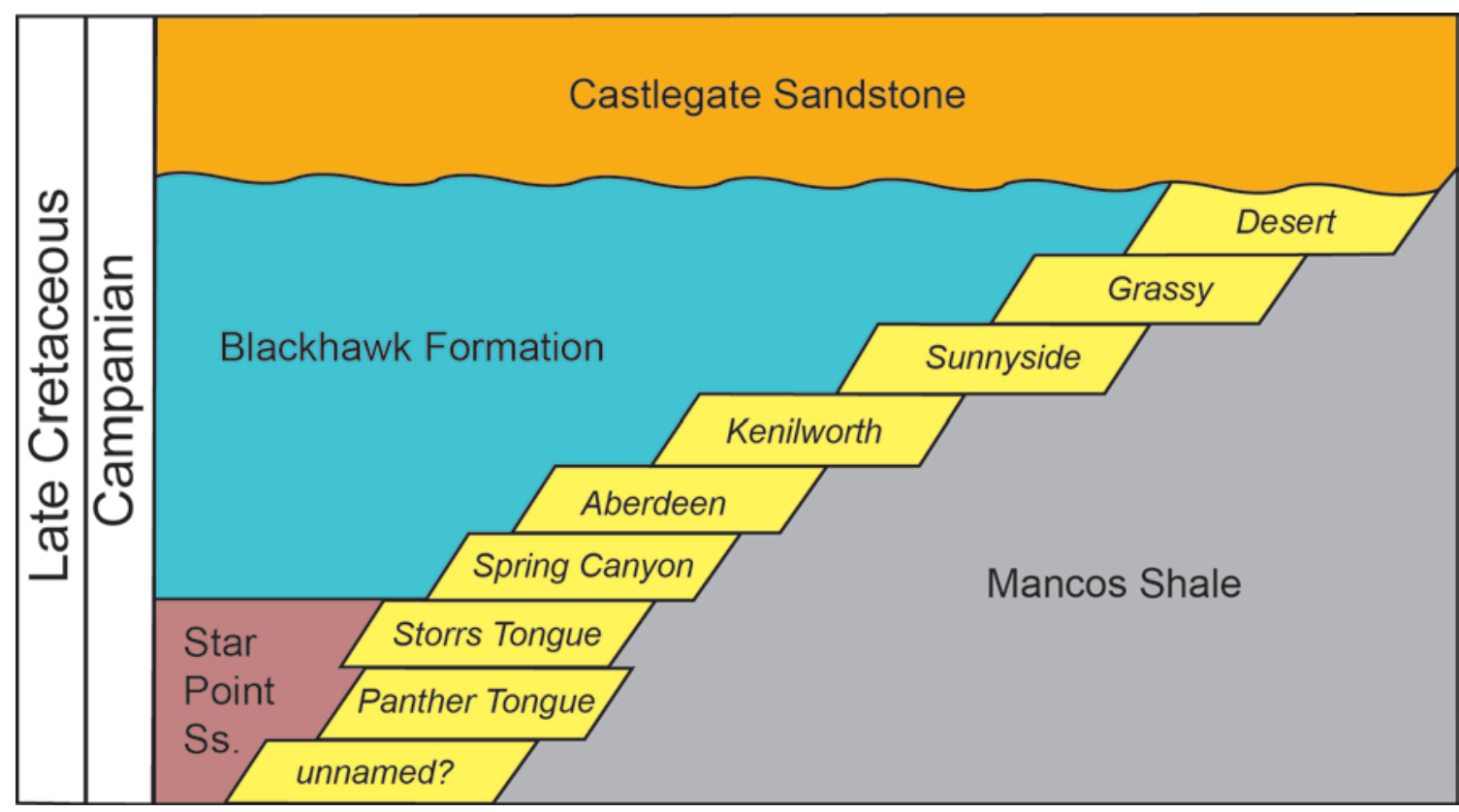

Figure 2. Generalized stratigraphic column showing the relative positions and ages of the units from which samples were taken for this study (modified from Young, 1955). Members of the Blackhawk Formation and Star Point Sandstone (in italics) are shown in yellow boxes. (Ss., sandstone)

\section{Methods and Results}

Petrologic and isotopic studies of the Blackhawk Formation and Star Point Sandstone included (1) petrography using standard and polished thin sections, (2) scanning electron microscopic (SEM) analyses, (3) X-ray diffraction analyses, and (4) carbon and oxygen isotopic determinations on calcite in coal samples and along fractures that cut sandstone samples. Samples for this study were obtained from both outcrops and drill cores. Fifteen outcrop sections were described and measured; they are shown on plate 1 (see table 1 for a listing of outcrop localities, locality abbreviations, and location information). The stratigraphic positions of samples collected for petrologic and geochemical studies are also shown in plate 1. Drill core location information is shown in table 2. Sample numbering is keyed to outcrop locality or the well from which the core was obtained. Herein, the first part of a sample number refers to the outcrop or core abbreviation, and the second part refers to the number designated in the measured section or depth for core samples. Numbers with the designation Kbh refer to samples from the Cretaceous Blackhawk Formation, whereas those designated Ksp are from the Cretaceous Star Point Sandstone. Several samples from the Gentile Wash locality were collected from parasequences in the Blackhawk Formation, and the numbering scheme for those samples also references the specific parasequence from which materials were collected (see plate 1). 
Table 1. Location information for outcrops from which samples of the Blackhawk Formation and (or) Star Point Sandstone were collected. See plate 1 for measured sections at these localities, including the stratigraphic position of samples. See figure 1 for a map view of these localities.

\begin{tabular}{|l|c|c|c|c|c|}
\hline \multirow{2}{*}{ Outcrop locality name } & \multirow{2}{*}{ Locality abbreviation } & \multicolumn{4}{c|}{ Outcrop location information } \\
\cline { 3 - 6 } & & Township & Range & Section & County \\
\hline Anderson Mine & $\mathrm{AM}$ & $19 \mathrm{~S}$ & $7 \mathrm{E}$ & 7 & Emery \\
\hline Biddlecomb Hollow & $\mathrm{BH}$ & $20 \mathrm{~S}$ & $6 \mathrm{E}$ & 3 & Emery \\
\hline Birch Spring & $\mathrm{BS}$ & $16 \mathrm{~S}$ & $7 \mathrm{E}$ & 26 & Emery \\
\hline Black Diamond Mine & $\mathrm{BDM}$ & $18 \mathrm{~S}$ & $6 \mathrm{E}$ & 12 & Emery \\
\hline Candland Mountain (partial section) & $\mathrm{CAN}$ & $14 \mathrm{~S}$ & $6 \mathrm{E}$ & 26 & Emery \\
\hline Candland Mountain (partial section) & $\mathrm{CAN}$ & $14 \mathrm{~S}$ & $6 \mathrm{E}$ & 23 & Emery \\
\hline Co-op Mine & $\mathrm{CM}$ & $16 \mathrm{~S}$ & $7 \mathrm{E}$ & 22 & Emery \\
\hline Electric Lake & $\mathrm{EL}$ & $14 \mathrm{~S}$ & $6 \mathrm{E}$ & 14 & Emery \\
\hline Gentile Wash & $\mathrm{GW}$ & $13 \mathrm{~S}$ & $9 \mathrm{E}$ & 12 & Carbon \\
\hline Interstate 70, mileage post 64 & $\mathrm{I70} / 64$ & $22 \mathrm{~S}$ & $2 \mathrm{E}$ & 15 & Emery \\
\hline Joes Valley Reservoir & $\mathrm{JVR}$ & $18 \mathrm{~S}$ & $6 \mathrm{E}$ & 5 & Emery \\
\hline Pleasant Creek & $\mathrm{PC}$ & $15 \mathrm{~S}$ & $5 \mathrm{E}$ & 11 & Sanpete \\
\hline Straight Canyon & $\mathrm{SC}$ & $18 \mathrm{~S}$ & $6 \mathrm{E}$ & 11 & Emery \\
\hline Suffco Mine & $\mathrm{SF}$ & $22 \mathrm{~S}$ & $4 \mathrm{E}$ & 12 & Emery \\
\hline Twelvemile Creek & TMC (TMB-b) & $19 \mathrm{~S}$ & $3 \mathrm{E}$ & 33 & Sanpete \\
\hline
\end{tabular}


Table 2. Location information for core samples of the Cretaceous Blackhawk Formation that were used in this study. Because depths at the Cyprus Coal core are indeterminate, core box number is given instead of sampling depth.

[API, American Petroleum Institute; ft, feet; do, same as above]

\begin{tabular}{|c|c|c|c|c|c|c|c|c|c|}
\hline \multirow{2}{*}{ Core name } & \multirow{2}{*}{$\begin{array}{c}\text { Core } \\
\text { abbreviation }\end{array}$} & \multicolumn{4}{|c|}{ Well location information } & \multirow{2}{*}{ Well API number } & \multirow{2}{*}{ Sample number } & \multirow{2}{*}{$\begin{array}{l}\text { Sample depth } \\
\text { (ft) }\end{array}$} & \multirow{2}{*}{ Sample type } \\
\hline & & Township & Range & Section & County & & & & \\
\hline \multirow[t]{5}{*}{ Cyprus Coal 1} & Cyprus Coal & $15 \mathrm{~S}$ & $8 \mathrm{E}$ & 18 & Carbon & $43-007-80000$ & Cyprus Coal 1 & Box 7 & coal \\
\hline & & & & & & & Cyprus Coal 2 & Box 6 & sandstone \\
\hline & & & & & & & Cyprus Coal 3 & Box 6 & sandstone \\
\hline & & & & & & & Cyprus Coal 4 & Box 4 & coal \\
\hline & & & & & & & Cyprus Coal 5 & Box 2 & coal \\
\hline \multirow[t]{2}{*}{$\begin{array}{l}\text { North Trough } \\
\text { Spring-10 }\end{array}$} & NTS & $14 \mathrm{~S}$ & $7 \mathrm{E}$ & 19 & Carbon & $43-007-80001$ & NTS-10-1 & 59 & coal \\
\hline & & & & & & & NTS-10-3 & 47.5 & coal \\
\hline \multirow[t]{10}{*}{$\begin{array}{l}\text { South Horn } \\
\quad \text { Mountain-1A }\end{array}$} & SHM-1A & $19 \mathrm{~S}$ & $6 \mathrm{E}$ & 15 & Emery & $43-015-80000$ & SHM-1A-1 & $1,332.5$ & sandstone \\
\hline & & & & & & & SHM-1A-2 & $1,324.0$ & sandstone \\
\hline & & & & & & & SHM-1A-3 & $1,317.5$ & sandstone \\
\hline & & & & & & & SHM-1A-4 & $1,305.0$ & coal \\
\hline & & & & & & & SHM-1A-5 & $1,296.5$ & coal \\
\hline & & & & & & & SHM-1A-6 & $1,273.0$ & sandstone \\
\hline & & & & & & & SHM-1A-7 & $1,269.5$ & fracture \\
\hline & & & & & & & SHM-1A-9 & $1,256.5$ & fracture \\
\hline & & & & & & & SHM-1A-11 & $1,251.0$ & sandstone \\
\hline & & & & & & & SHM-1A-12 & $1,244.5$ & fracture \\
\hline \multirow[t]{2}{*}{$\begin{array}{l}\text { South Horn } \\
\text { Mountain-2A }\end{array}$} & SHM-2A & $19 \mathrm{~S}$ & $6 \mathrm{E}$ & 9 & Emery & $43-015-80002$ & SHM-2A-1 & $1,359.0$ & sandstone \\
\hline & & & & & & & SHM-2A-2 & $1,343.0$ & sandstone \\
\hline \multirow[t]{4}{*}{$\begin{array}{l}\text { South Horn } \\
\quad \text { Mountain-2 }\end{array}$} & SHM-2 & $19 \mathrm{~S}$ & $6 \mathrm{E}$ & 11 & do & $43-015-80000$ & SHM-2-1 & $1,118.5$ & sandstone \\
\hline & & & & & & & SHM-2-2 & $1,113.0$ & sandstone \\
\hline & & & & & & & SHM-2-3 & $1,110.0$ & coal \\
\hline & & & & & & & SHM-2-4 & $1,095.0$ & sandstone \\
\hline
\end{tabular}


Table 2. Location information for core samples of the Cretaceous Blackhawk Formation that were used in this study. Because depths at the Cyprus Coal core are indeterminate, core box number is given instead of sampling depth.-Continued

[API, American Petroleum Institute; ft, feet; do, same as above]

\begin{tabular}{|c|c|c|c|c|c|c|c|c|c|}
\hline \multirow{2}{*}{ Core name } & \multirow{2}{*}{$\begin{array}{c}\text { Core } \\
\text { abbreviation }\end{array}$} & \multicolumn{4}{|c|}{ Well location information } & \multirow{2}{*}{ Well API number } & \multirow{2}{*}{ Sample number } & \multirow{2}{*}{$\begin{array}{l}\text { Sample depth } \\
\text { (ft) }\end{array}$} & \multirow{2}{*}{ Sample type } \\
\hline & & Township & Range & Section & County & & & & \\
\hline & & & & & & & SHM-2-6 & $1,069.0$ & Coal \\
\hline & & & & & & & SHM-2-7 & $1,059.5$ & coal \\
\hline & & & & & & & SHM-2-8 & $1,055.0$ & coal \\
\hline \multirow[t]{6}{*}{$\begin{array}{l}\text { South Horn } \\
\text { Mountain-5 }\end{array}$} & SHM-5 & $19 \mathrm{~S}$ & $6 \mathrm{E}$ & 22 & do & $43-015-80001$ & SHM-5-1 & $1,035.0$ & sandstone \\
\hline & & & & & & & SHM-5-2 & $1,028.5$ & sandstone \\
\hline & & & & & & & SHM-5-3 & $1,015.5$ & sandstone \\
\hline & & & & & & & SHM-5-4 & $1,007.5$ & sandstone \\
\hline & & & & & & & SHM-5-5 & $1,003.0$ & sandstone \\
\hline & & & & & & & SHM-5-6 & $1,001.0$ & coal \\
\hline \multirow[t]{3}{*}{$\begin{array}{l}\text { South Horn } \\
\text { Mountain-13 }\end{array}$} & SHM-13 & $19 \mathrm{~S}$ & $6 \mathrm{E}$ & 26 & do & $43-015-80003$ & SHM-13-2 & $1,116.0$ & coal \\
\hline & & & & & & & SHM-13-3 & $1,113.0$ & sandstone \\
\hline & & & & & & & SHM-13-4 & $1,111.0$ & sandstone \\
\hline \multirow[t]{4}{*}{$\begin{array}{l}\text { South Horn } \\
\text { Mountain-14 }\end{array}$} & SHM-14 & $19 \mathrm{~S}$ & $6 \mathrm{E}$ & 35 & do & $43-015-80004$ & SHM-14-1 & 987.5 & sandstone \\
\hline & & & & & & & SHM-14-2 & 983.0 & sandstone \\
\hline & & & & & & & SHM-14-3 & 982.0 & sandstone \\
\hline & & & & & & & SHM-14-4 & 941.5 & coal \\
\hline $\begin{array}{l}\text { South Horn } \\
\text { Mountain-15 }\end{array}$ & SHM-15 & $19 \mathrm{~S}$ & $6 \mathrm{E}$ & 21 & Emery & $43-015-80005$ & SHM-15-1 & 1,118 & sandstone \\
\hline \multirow[t]{7}{*}{ Trail Mountain-7 } & TM-7 & $17 \mathrm{~S}$ & $6 \mathrm{E}$ & 27 & Emery & $43-015-80933$ & TM-7-1 & $2,498.5$ & sandstone \\
\hline & & & & & & & TM-7-2 & $2,428.0$ & sandstone \\
\hline & & & & & & & TM-7-6 & $2,368.5$ & sandstone \\
\hline & & & & & & & TM-7-7 & $2,341.5$ & sandstone \\
\hline & & & & & & & TM-7-8 & $2,302.5$ & sandstone \\
\hline & & & & & & & TM-7-10 & $2,242.5$ & sandstone \\
\hline & & & & & & & TM-7-11 & $2,225.5$ & sandstone \\
\hline
\end{tabular}


Table 2. Location information for core samples of the Cretaceous Blackhawk Formation that were used in this study. Because depths at the Cyprus Coal core are indeterminate, core box number is given instead of sampling depth.-Continued

[API, American Petroleum Institute; ft, feet; do, same as above]

\begin{tabular}{|c|c|c|c|c|c|c|c|c|c|}
\hline \multirow{2}{*}{ Core name } & \multirow{2}{*}{$\begin{array}{c}\text { Core } \\
\text { abbreviation }\end{array}$} & \multicolumn{4}{|c|}{ Well location information } & \multirow{2}{*}{ Well API number } & \multirow{2}{*}{ Sample number } & \multirow{2}{*}{$\begin{array}{l}\text { Sample depth } \\
\text { (ft) }\end{array}$} & \multirow{2}{*}{ Sample type } \\
\hline & & Township & Range & Section & County & & & & \\
\hline & & & & & & & TM-7-12 & $2,195.0$ & coal \\
\hline & & & & & & & TM-7-13 & $2,190.0$ & sandstone \\
\hline & & & & & & & TM-7-16 & $2,119.5$ & fracture \\
\hline & & & & & & & TM-7-17 & $2,081.0$ & coal \\
\hline & & & & & & & TM-7-19 & $2,052.0$ & sandstone \\
\hline & & & & & & & TM-7-20 & $2,012.0$ & coal \\
\hline & & & & & & & TM-7-21 & $1,953.5$ & sandstone \\
\hline & & & & & & & TM-7-23 & $1,953.0$ & sandstone \\
\hline & & & & & & & TM-7-24 & $1,935.0$ & sandstone \\
\hline & & & & & & & TM-7-25 & $1,925.7$ & coal \\
\hline & & & & & & & TM-7-26 & $1,876.0$ & sandstone \\
\hline & & & & & & & TM-7-27 & $1,868.0$ & sandstone \\
\hline & & & & & & & TM-7-29 & $1,800.5$ & sandstone \\
\hline & & & & & & & TM-7-30 & $1,778.5$ & sandstone \\
\hline & & & & & & & TM-7-31 & $1,758.0$ & coal \\
\hline & & & & & & & TM-7-32 & $1,697.0$ & fracture \\
\hline & & & & & & & TM-7-33 & $1,675.0$ & sandstone \\
\hline & & & & & & & TM-7-34 & $1,640.6$ & coal \\
\hline & & & & & & & TM-7-35 & $1,615.0$ & sandstone \\
\hline & & & & & & & TM-7-36 & $1,599.0$ & sandstone \\
\hline & & & & & & & TM-7-37 & $1,590.0$ & sandstone \\
\hline & & & & & & & TM-7-39 & $1,553.0$ & sandstone \\
\hline & & & & & & & TM-7-40 & $1,535.5$ & sandstone \\
\hline & & & & & & & TM-7-41 & $1,510.0$ & sandstone \\
\hline & & & & & & & TM-7-42 & $1,506.0$ & sandstone \\
\hline
\end{tabular}


Petrographic analyses provided data concerning the modal composition of sandstones in the Blackhawk and Star Point, as well as the fundamental relations that facilitated construction of a paragenetic sequence of alterations for the sandstones (fig. 3). Rock chips used in making thin sections $(n=238)$ were impregnated with blue epoxy to better observe the quantity and distribution of porosity. The thin sections were stained with (1) alizarin red-S and potassium ferricyanide to assist in carbonate mineral compositional determination (after Dickson, 1966) and (2) sodium cobaltinitrite to identify potassium feldspar. Point counts (300 points/thin section) were performed on representative $(n=38)$ thin sections of sandstones, and the count data were converted to volume percent (see table 3 ).

PARAGENETIC SEQUENCE OF ALTERATIONS

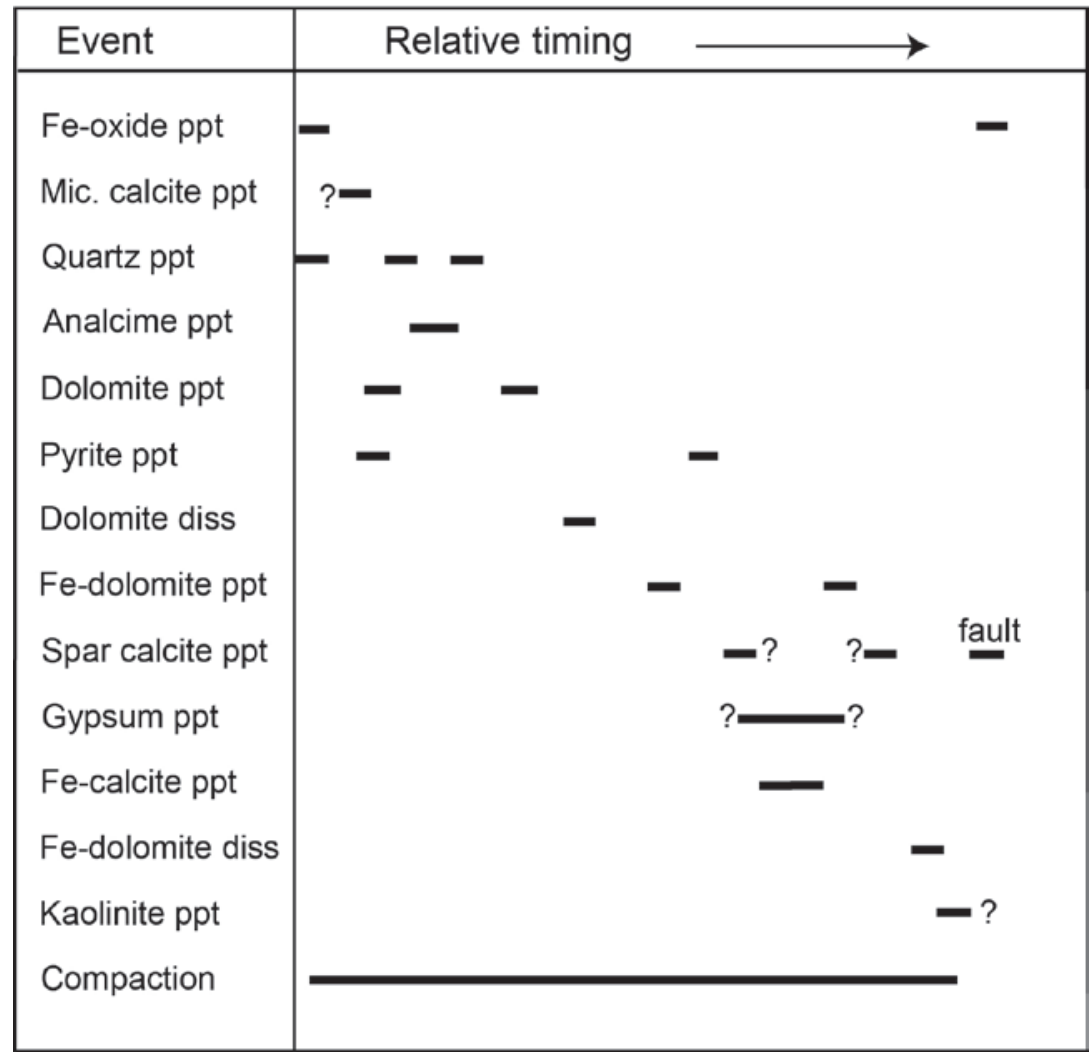

Figure 3. Summary of the paragenetic sequence of alterations in sandstone beds in the Blackhawk Formation and Star Point Sandstone. Arrow points in direction of decreasing age. (ppt, precipitation; Fe, iron; mic., micritic; diss, dissolution; fault, calcite along faults) 
Table 3. Point count data (converted to volume percent) for sandstones from either the Blackhawk Formation ("bh" in sample number) or Star Point Sandstone ("sp" in sample number). See tables 1 and 2 for key to outcrop and core abbreviations and locations, respectively.

$[\%$, percent; RF, rock fragments; Misc., miscellaneous detrital grains (such as heavy minerals); Non-Fe Cal., non-ferroan calcite; Fe-calcite, ferroan calcite; Fedolomite, ferroan dolomite; OV, overgrowth; Fe-oxide, iron oxide]

\begin{tabular}{|c|c|c|c|c|c|c|c|c|c|c|c|c|c|c|}
\hline \multirow{2}{*}{$\begin{array}{l}\text { Sample } \\
\text { number }\end{array}$} & \multirow{2}{*}{$\begin{array}{l}\text { Formation } \\
\text { sampled }\end{array}$} & \multicolumn{5}{|c|}{$\begin{array}{l}\text { Detrital grains } \\
\text { (volume \%) }\end{array}$} & \multicolumn{5}{|c|}{$\begin{array}{l}\text { Authigenic phases } \\
\text { (volume \%) }\end{array}$} & \multicolumn{2}{|c|}{$\begin{array}{l}\text { Porosity } \\
\text { (volume \%) }\end{array}$} & \multirow{2}{*}{ Total \% } \\
\hline & & Quartz & Feldspar & RF & Misc. & $\begin{array}{c}\text { Non-Fe } \\
\text { Cal. }\end{array}$ & $\begin{array}{c}\mathrm{Fe}- \\
\text { calcite }\end{array}$ & Dolomite & $\begin{array}{c}\text { Fe- } \\
\text { dolomite }\end{array}$ & $\begin{array}{l}\text { Quartz } \\
\text { OV }\end{array}$ & $\begin{array}{c}\mathrm{Fe}- \\
\text { oxide }\end{array}$ & Primary & Secondary & \\
\hline AM-Kbh-4 & Blackhawk & 59 & 0 & 25 & 0 & 2 & 0 & 0 & 0 & 1 & 0 & 12 & 0 & 100 \\
\hline AM-Kbh-8 & Blackhawk & 62 & 1 & 21 & 3 & 0 & 0 & 0 & 0 & 1 & 0 & 10 & 0 & 100 \\
\hline BDM-Kbh-1 & Blackhawk & 56 & 4 & 32 & 0 & 4 & 0 & 0 & 0 & 0 & 2 & 3 & 0 & 100 \\
\hline BDM-Kbh-11 & Blackhawk & 51 & 0 & 28 & 2 & 6 & 0 & 0 & 0 & 3 & 1 & 8 & 1 & 100 \\
\hline BDM-Kbh-3 & Blackhawk & 51 & 0 & 30 & 2 & 7 & 0 & 0 & 0 & 1 & 0 & 8 & 1 & 100 \\
\hline BH-Kbh-1 & Blackhawk & 56 & 0 & 26 & 0 & 5 & 0 & 1 & 0 & 2 & 2 & 7 & 1 & 100 \\
\hline BH-Ksp-1 & Star Point & 50 & 7 & 24 & 4 & 0 & 2 & 1 & 0 & 0 & 1 & 10 & 0 & 100 \\
\hline BH-Ksp-10 & Star Point & 40 & 1 & 36 & 0 & 0 & 0 & 0 & 0 & 0 & 1 & 20 & 1 & 100 \\
\hline BH-Ksp-8 & Star Point & 48 & 1 & 24 & 1 & 3 & 0 & 1 & 0 & 1 & 1 & 20 & 1 & 100 \\
\hline BS-Kbh-3 & Blackhawk & 53 & 0 & 32 & 0 & 7 & 0 & 0 & 0 & 1 & 0 & 6 & 1 & 100 \\
\hline BS-Ksp-1 & Star Point & 55 & 1 & 28 & 0 & 1 & 1 & 0 & 1 & 2 & 0 & 11 & 0 & 100 \\
\hline BS-Ksp-13 & Star Point & 58 & 1 & 25 & 0 & 1 & 1 & 0 & 0 & 1 & 0 & 12 & 0 & 100 \\
\hline BS-Ksp-6 & Star Point & 49 & 3 & 34 & 0 & 10 & 0 & 0 & 0 & 1 & 0 & 4 & 0 & 100 \\
\hline GW-Kbhhe-1 & Blackhawk & 72 & 3 & 8 & 0 & 7 & 0 & 0 & 0 & 1 & 1 & 8 & 1 & 100 \\
\hline GW-Kbhsb-3 & Blackhawk & 58 & 0 & 23 & 1 & 1 & 3 & 0 & 2 & 1 & 3 & 7 & 0 & 100 \\
\hline I70/64-Kbh-1 & Blackhawk & 46 & 3 & 30 & 9 & 2 & 9 & 0 & 0 & 0 & 0 & 0 & 0 & 100 \\
\hline I70/64-Kbh-5 & Blackhawk & 62 & 1 & 12 & 7 & 0 & 0 & 0 & 0 & 1 & 10 & 7 & 0 & 100 \\
\hline I70/64-Kbh-8 & Blackhawk & 58 & 1 & 19 & 0 & 0 & 0 & 1 & 1 & 0 & 3 & 16 & 2 & 100 \\
\hline SHM-15-1 & Blackhawk & 65 & 0 & 11 & 0 & 0 & 0 & 0 & 0 & 2 & 0 & 20 & 1 & 100 \\
\hline
\end{tabular}


Table 3. Point count data (converted to volume percent) for sandstones from either the Blackhawk Formation ("bh" in sample number) or Star Point Sandstone ("sp" in sample number). See tables 1 and 2 for key to outcrop and core abbreviations and locations, respectively.-Continued

$[\%$, percent; RF, rock fragments; Misc., miscellaneous detrital grains (such as heavy minerals); Non-Fe Cal., non-ferroan calcite; Fe-calcite, ferroan calcite; Fedolomite, ferroan dolomite; OV, overgrowth; Fe-oxide, iron oxide]

\begin{tabular}{|c|c|c|c|c|c|c|c|c|c|c|c|c|c|c|}
\hline \multirow{2}{*}{$\begin{array}{l}\text { Sample } \\
\text { number }\end{array}$} & \multirow{2}{*}{$\begin{array}{l}\text { Formation } \\
\text { sampled }\end{array}$} & \multicolumn{5}{|c|}{$\begin{array}{l}\text { Detrital grains } \\
\text { (volume \%) }\end{array}$} & \multicolumn{5}{|c|}{$\begin{array}{l}\text { Authigenic phases } \\
\text { (volume \%) }\end{array}$} & \multicolumn{2}{|c|}{$\begin{array}{c}\text { Porosity } \\
\text { (volume \%) }\end{array}$} & \multirow{2}{*}{ Total \% } \\
\hline & & Quartz & Feldspar & RF & Misc. & $\begin{array}{c}\text { Non-Fe } \\
\text { Cal. }\end{array}$ & $\begin{array}{c}\text { Fe- } \\
\text { calcite }\end{array}$ & Dolomite & $\begin{array}{c}\text { Fe- } \\
\text { dolomite }\end{array}$ & $\begin{array}{c}\text { Quartz } \\
\text { OV }\end{array}$ & $\begin{array}{c}\begin{array}{c}\text { Fe- } \\
\text { oxide }\end{array} \\
\text { onde }\end{array}$ & Primary & Secondary & \\
\hline SHM-1A-1 & Blackhawk & 52 & 0 & 27 & 0 & 2 & 0 & 0 & 0 & 4 & 0 & 15 & 1 & 100 \\
\hline SHM-1A-11 & Blackhawk & 48 & 0 & 33 & 0 & 0 & 12 & 2 & 4 & 0 & 0 & 0 & 0 & 100 \\
\hline SHM-1A-2 & Blackhawk & 67 & 0 & 13 & 0 & 0 & 0 & 0 & 0 & 3 & 0 & 15 & 1 & 100 \\
\hline SHM-1A-3 & Blackhawk & 67 & 0 & 4 & 0 & 0 & 0 & 0 & 0 & 1 & 14 & 13 & 1 & 100 \\
\hline SHM-1A-6 & Blackhawk & 62 & 0 & 11 & 0 & 0 & 11 & 5 & 4 & 5 & 0 & 2 & 0 & 100 \\
\hline SHM-2-2 & Blackhawk & 74 & 0 & 9 & 0 & 0 & 0 & 0 & 0 & 2 & 8 & 7 & 1 & 100 \\
\hline SHM-2A-1 & Blackhawk & 47 & 1 & 29 & 0 & 4 & 2 & 0 & 1 & 2 & 0 & 15 & 1 & 100 \\
\hline SHM-2A-2 & Blackhawk & 43 & 0 & 37 & 0 & 5 & 0 & 0 & 2 & 2 & 0 & 11 & 0 & 100 \\
\hline SHM-5-3 & Blackhawk & 57 & 1 & 18 & 0 & 0 & 0 & 0 & 0 & 2 & 0 & 21 & 0 & 100 \\
\hline SHM-5-4 & Blackhawk & 68 & 0 & 8 & 1 & 0 & 0 & 0 & 0 & 3 & 3 & 16 & 1 & 100 \\
\hline SHM-5-5 & Blackhawk & 70 & 0 & 5 & 0 & 0 & 0 & 0 & 0 & 2 & 8 & 15 & 1 & 100 \\
\hline TMC-b-Kbh-1 & Blackhawk & 69 & 1 & 20 & 7 & 0 & 0 & 0 & 0 & 0 & 0 & 3 & 1 & 100 \\
\hline TMC-b-Kbh-2 & Blackhawk & 71 & 1 & 9 & 4 & 0 & 0 & 0 & 0 & 0 & 0 & 15 & 1 & 100 \\
\hline TMC-b-Kbh-4 & Blackhawk & 67 & 1 & 16 & 3 & 0 & 0 & 0 & 0 & 0 & 0 & 13 & 0 & 100 \\
\hline TMC-Kbh-5 & Blackhawk & 65 & 1 & 9 & 4 & 0 & 0 & 0 & 0 & 4 & 0 & 16 & 0 & 100 \\
\hline TMC-Kbh-7 & Blackhawk & 72 & 0 & 13 & 14 & 0 & 0 & 0 & 0 & 0 & 0 & 2 & 0 & 100 \\
\hline TMC-Kbh-8 & Blackhawk & 70 & 1 & 11 & 8 & 0 & 2 & 0 & 0 & 2 & 0 & 4 & 1 & 100 \\
\hline TMC-Kbh-9 & Blackhawk & 79 & 0 & 7 & 2 & 0 & 0 & 0 & 0 & 3 & 0 & 8 & 1 & 100 \\
\hline
\end{tabular}


Paragenetic relations between alteration events in sandstones (see fig. 3) were refined through SEM investigations, and the SEM was also used to determine the composition of authigenic minerals. Rock chips of selected samples were coated with gold for SEM viewing and analyses. The SEM was equipped with an energy dispersive analyzer of X-rays, which provided information concerning the elements within the minerals being studied (after Goldstein and others, 1981). In addition, SEM studies were undertaken on 18 polished thin sections to enhance our understanding of the distribution of elements in detrital and authigenic minerals.

$\mathrm{X}$-ray diffraction (XRD) analysis, using standard XRD techniques with $\mathrm{CuK}_{\alpha}$ radiation, was performed on material collected from coal cleats or fractures. Minerals removed from coals of the Blackhawk Formation were from apertures along one of two types of cleats, either face cleats or butt cleats (cleat terminology after Laubach and others, 1998). Cleats are defined as those fractures approximately normal to bedding and approximately perpendicular to each other. Much of the mineral material used for this study was likely from face cleats, in that these were the cleats that were more prominent and into which the subordinate butt cleats commonly terminated. Minerals were also obtained from fractures that cut across beds, including coals, sandstones, and siltstones. Cleats and fracture surfaces were exposed by pulling rock samples apart, and the minerals present on these surfaces were then removed with a scalpel and tweezers. The minerals that were identified in the cleats and fractures are summarized in table 4. 
Table 4. Blackhawk Formation samples from which material was obtained from apertures (either cleats or fractures) and analyzed by X-ray diffraction and (or) scanning electron microscope.

[Fe, iron; $\mathrm{NaCl}$, sodium chloride; coarse, coarse crystal size; fine, fine crystal size. See table 1 for location of AM, GW, and I70/64 sites and table 2 for location of Cyprus Coal, NTS, SHM-1A, SHM-2, SHM-5, SHM-13, SHM-14, and TM cores]

\begin{tabular}{|c|c|c|c|c|c|c|c|c|c|c|c|c|c|}
\hline \multirow{2}{*}{$\begin{array}{l}\text { Sample } \\
\text { number }\end{array}$} & \multirow{2}{*}{$\begin{array}{l}\text { Source of } \\
\text { mineral(s) }\end{array}$} & \multicolumn{12}{|c|}{ Mineral(s) present } \\
\hline & & Quartz & $\begin{array}{l}\text { Non-Fe } \\
\text { calcite }\end{array}$ & $\begin{array}{c}\text { Non-Fe } \\
\text { dolomite }\end{array}$ & $\begin{array}{c}\text { Fe- } \\
\text { dolomite }\end{array}$ & Ankerite & Analcime & $\mathrm{NaCl}$ & Gypsum & Kaolinite & Illite & Pyrite & Barite \\
\hline AM-Kbh-1 & Cleat & & & & & & & & $\mathrm{X}$ & & & & \\
\hline BDM-Kbh-10 & Cleat & & & & & & $\mathrm{X}$ & & $\mathrm{X}$ & & & & \\
\hline BDM-Kbh-5 & Cleat & & & & & & & & $\mathrm{X}$ & & & & \\
\hline BDM-Kbh-7 & Cleat & $\mathrm{X}$ & $\mathrm{X}$ & & & & $\mathrm{X}$ & & & $\mathrm{X}$ & & & \\
\hline BH-Ksp-13 & Cleat & $\mathrm{X}$ & & & & & & & & $\mathrm{X}$ & & & \\
\hline CM-Kbh-3 & Cleat & & $\mathrm{X}$ & & & & & & & $\mathrm{X}$ & & & \\
\hline Cyprus Coal 1 & Cleat & & & $\mathrm{X}$ & & & $\mathrm{X}$ & & & & & & \\
\hline Cyprus Coal 4 & Cleat & & $\mathrm{X}$ & $\mathrm{X}$ & & $\mathrm{X}$ & $\mathrm{X}$ & & & & & $\mathrm{X}$ & \\
\hline Cyprus Coal 5 & Cleat & & $\mathrm{X}$ & & & & & & & & & $\mathrm{X}$ & \\
\hline GW-Kbhhp-3 & Cleat & & $\mathrm{X}$ & $\mathrm{X}$ & & & & & $\mathrm{X}$ & $\mathrm{X}$ & & & \\
\hline GW-Kbhsb-11 & Cleat & & & & & & & $\mathrm{X}$ & $\mathrm{X}$ & $\mathrm{X}$ & & & \\
\hline I70/64 Kbh-4 & Cleat & $\mathrm{X}$ & & & & & & & $\mathrm{X}$ & $\mathrm{X}$ & & & \\
\hline NTS-10-1 & Cleat & & $\mathrm{X}$ & & & & & & & & & & \\
\hline NTS-10-3 & Cleat & & $\mathrm{X}$ & & & & & & & $\mathrm{X}$ & & $\mathrm{X}$ & \\
\hline SC-Kbh-2 & Cleat & & & & & & $\mathrm{X}$ & & & $X$ & & & \\
\hline SHM-1A-4 & Cleat & & $\mathrm{X}$ & & & & & & & & & & \\
\hline SHM-1A-5 & Cleat & & & & & & $\mathrm{X}$ & & & & & $\mathrm{X}$ & \\
\hline SHM-1A-7 & Fracture & & $\mathrm{X}$ & & & & & & & & & & \\
\hline SHM-1A-9 & Fracture & & $\mathrm{X}$ & $\mathrm{X}$ & & $\mathrm{X}$ & & & & & & & \\
\hline
\end{tabular}


Table 4. Blackhawk Formation samples from which material was obtained from apertures (either cleats or fractures) and analyzed by X-ray diffraction and (or) scanning electron microscope.-Continued

[Fe, iron; $\mathrm{NaCl}$, sodium chloride; coarse, coarse crystal size; fine, fine crystal size. See table 1 for location of AM, GW, and I70/64 sites and table 2 for location of Cyprus Coal, NTS, SHM-1A, SHM-2, SHM-5, SHM-13, SHM-14, and TM cores]

\begin{tabular}{|c|c|c|c|c|c|c|c|c|c|c|c|c|c|}
\hline \multirow[b]{2}{*}{$\begin{array}{l}\text { Sample } \\
\text { number }\end{array}$} & \multirow[b]{2}{*}{$\begin{array}{l}\text { Source of } \\
\text { mineral(s) }\end{array}$} & \multicolumn{12}{|c|}{ Mineral(s) present } \\
\hline & & Quartz & $\begin{array}{l}\text { Non-Fe } \\
\text { calcite }\end{array}$ & $\begin{array}{c}\text { Non-Fe } \\
\text { dolomite }\end{array}$ & $\begin{array}{c}\text { Fe- } \\
\text { dolomite }\end{array}$ & Ankerite & Analcime & $\mathrm{NaCl}$ & Gypsum & Kaolinite & Illite & Pyrite & Barite \\
\hline SHM-1A-12 coarse & Fracture & & $\mathrm{X}$ & & & & & & & & & & \\
\hline SHM-1A-12 fine & Fracture & & $\mathrm{X}$ & & & & & & & & & & \\
\hline SHM-2-3 & Cleat & & & & & & $\mathrm{X}$ & & & & & $\mathrm{X}$ & \\
\hline SHM-2-6 & Cleat & & $\mathrm{X}$ & & & & & & & & & & \\
\hline SHM-2-7 & Cleat & & $X$ & & & & $\mathrm{X}$ & & & & & $\mathrm{X}$ & \\
\hline SHM-2-8 & Cleat & & & & & & & & & & & $\mathrm{X}$ & $\mathrm{X}$ \\
\hline SHM-5-6 & Cleat & & & & & & & & & $\mathrm{X}$ & & & $\mathrm{X}$ \\
\hline SHM-13-2 & Cleat & & $\mathrm{X}$ & $\mathrm{X}$ & & $\mathrm{X}$ & $\mathrm{X}$ & & & & & $\mathrm{X}$ & \\
\hline SHM-13-3 & Fracture & & & $\mathrm{X}$ & & & & & & $\mathrm{X}$ & & & $\mathrm{X}$ \\
\hline SHM-14-4 & Cleat & & $\mathrm{X}$ & & $\mathrm{X}$ & & $\mathrm{X}$ & & $\mathrm{X}$ & & & $\mathrm{X}$ & \\
\hline TM-7-12 & Cleat & $X$ & & & & & & & & & & & \\
\hline TM-7-16 fine & Fracture & & $\mathrm{X}$ & & & & & & & & & & \\
\hline TM-7-16 coarse & Fracture & & $\mathrm{X}$ & & & & & & & & & & \\
\hline TM-7-17 & Cleat & & $\mathrm{X}$ & & & & & & $\mathrm{X}$ & & & $\mathrm{X}$ & \\
\hline TM-7-20 & Cleat & & $\mathrm{X}$ & & & & $\mathrm{X}$ & & & & & & \\
\hline TM-7-25 & Cleat & & $\mathrm{X}$ & $\mathrm{X}$ & & & & & & & & & \\
\hline TM-7-31 & Cleat & & $\mathrm{X}$ & & & & & & $\mathrm{X}$ & $X$ & & $X$ & \\
\hline TM-7-32 & Fracture & $X$ & & & & & & & & & & & $X$ \\
\hline TM-7-34 & Cleat & & & & & & & & & $X$ & & & $X$ \\
\hline
\end{tabular}


Where sufficient calcite was present along a cleat or fracture, the carbon and oxygen isotopic composition of the calcite was determined. Isotopic determinations were performed on 20 samples of fracture-fill calcite from various localities and 4 samples of authigenic calcite that was present as a cleat-filling mineral. Isotopic measurements were made on the $\mathrm{CO}_{2}$ that was produced upon reaction with phosphoric acid at $25^{\circ} \mathrm{C}$ during the first 6 hours. The isotopic data (table 5) are reported using the delta notation $\left(\delta^{13} \mathrm{C}\right.$ and $\left.\delta^{18} \mathrm{O}\right)$ referenced to the PDB standard (Belemnitella americana from the Upper Cretaceous Peedee Formation in South Carolina). The carbon and oxygen isotopic data presented in this report were calculated as follows:

$$
\begin{aligned}
& \left.\delta^{13} \mathrm{C}=\left(\left(R_{\text {sample }}\right) / R_{\text {standard }}\right)-1\right) \cdot 1000 \text { per mil }(\%) \\
& \left.\delta^{18} \mathrm{O}=\left(\left(R_{\text {sample }}\right) / R_{\text {standard }}\right)-1\right) \cdot 1000 \text { per mil }(\%)
\end{aligned}
$$

where

$$
R \quad \text { is }{ }^{13} \mathrm{C} /{ }^{12} \mathrm{C} \text { or }{ }^{18} \mathrm{O} /{ }^{16} \mathrm{O} \text {. }
$$

Oxygen isotopes are also presented in standard mean ocean water (SMOW) notation in table 5; SMOW values were determined by converting PDB values using the relationship $\delta_{\mathrm{SMOW}}=\left(\delta_{\mathrm{PDB}}+29.94\right) / 0.97006$.

Table 5. Carbon and oxygen isotopic composition of calcite from either cleats or fractures in the Blackhawk Formation (Kbh in sample number) or Star Point Sandstone (Ksp in sample number). See

\begin{tabular}{|c|c|c|c|c|}
\hline Sample number & Mineral occurrence & $\delta^{13} C_{(\mathrm{PDB})}$ & $\delta^{18} O_{(\mathrm{PDB})}$ & $\delta^{18} O_{\text {(SMOW) }}$ \\
\hline CM-Kbh-3 & Cleat & -8.497 & -17.406 & 12.966 \\
\hline Cyprus Coal 4 & Cleat & -3.258 & -14.079 & 16.396 \\
\hline Cyprus Coal 5 & Cleat & -5.993 & -19.578 & 10.726 \\
\hline TM-7-31 & Cleat & 1.171 & -18.069 & 12.282 \\
\hline BS-Kbh-3 edge & Fracture, adjacent to sandstone & -8.339 & -14.007 & 16.470 \\
\hline BS-Kbh-3 edge (replicate) & Fracture, adjacent to sandstone & -8.355 & -13.972 & 16.507 \\
\hline BS-Kbh-3 middle & Fracture, adjacent to "edge" & -7.719 & -13.846 & 16.636 \\
\hline BS-Kbh-3-center & Fracture, central part of fracture & -8.237 & -13.814 & 16.669 \\
\hline BS-Ksp-2A & Fracture & -6.857 & -16.661 & 13.734 \\
\hline BS-Ksp-2B & Fracture & -6.691 & -16.977 & 13.408 \\
\hline JVR-Kbh-1 center & Fracture, central part of fracture & -2.177 & -13.468 & 17.025 \\
\hline JVR-Kbh-1 middle & Fracture, adjacent to "edge" & -0.101 & -12.847 & 17.666 \\
\hline JVR-Kbh-1 edge & Fracture, adjacent to sandstone & -0.310 & -13.020 & 17.488 \\
\hline JVR-Kbh-2 center & Fracture, central part of fracture & -3.932 & -13.560 & 16.931 \\
\hline JVR-Kbh-2 middle & Fracture, adjacent to "edge" & -3.163 & -13.761 & 16.723 \\
\hline JVR-Kbh-2 edge & Fracture, adjacent to sandstone & -1.834 & -13.767 & 16.718 \\
\hline SHM-1A-12 coarse & Fracture, coarse crystals & -1.723 & -19.167 & 11.151 \\
\hline SHM-1A-12 fine & Fracture, fine crystals & -1.725 & -19.111 & 11.208 \\
\hline SHM-1A-4 & Fracture & 0.651 & -18.894 & 11.432 \\
\hline SHM-1A-7 & Fracture & -0.901 & -18.463 & 11.876 \\
\hline SHM-1A-9 & Fracture & -1.790 & -18.750 & 11.580 \\
\hline SHM-2-7 & Fracture & -4.294 & -15.092 & 15.351 \\
\hline TM-7-16 coarse & Fracture, coarse crystals & -1.724 & -16.096 & 14.317 \\
\hline TM-7-16 fine & Fracture, fine crystals & -1.796 & -17.218 & 13.160 \\
\hline
\end{tabular}
tables 1 and 2 for outcrop and core locations, respectively.

[PDB, Pee Dee Belemnite standard; SMOW, standard mean ocean water] 


\section{Acknowledgments}

The authors are grateful to the Utah Geological Survey for access to the cores from which samples were used in this study, and we benefited from conversations with Dave Tabet and Jeff Quick (both Utah Geological Survey), as well as Chris Cravitz (formerly with Suffco Mines) regarding our work. At the U.S. Geological Survey, we thank Jim Cathcart Jr. for the XRD analyses, Mary Ellen Benson for data compilation, Steve Cazenave for drafting plate 1, and Stephen Santus for additional drafting assistance. Reviews by Janet Pitman, Michael Brownfield, Dick Keefer, and Tom Judkins improved this manuscript and we are grateful for their comments and suggestions.

\section{References}

Dickson, J.A.D., 1966, Carbonate identification and genesis as revealed by staining: Journal of Sedimentary Petrology, v. 36, p. 491-505.

Ely, F.G., and Barnhart, D.H., 1963, Coal ash-Its effects on boiler availability, in Lawry, H.H., ed., Chemistry of coal utilization-Supplementary volume: New York, John Wiley and Sons, p. 820-891.

Finkelman, R.B., 1988, Analcime in coals of the Wasatch Plateau, Utah-Geological and technical significance, in Carter, L.M.H., ed., USGS research on energy resources, 1988: U.S. Geological Survey Circular 1025, p. 16-17.

Finkelman, R.B., 1991, Distribution and significance of analcime in Wasatch Plateau coals, in Chidsey, T.C., ed., Geology of east-central Utah: Utah Geological Association Publication 19, p. 173-181.

Finkelman, R.B., and Dulong, F.T., 1989, Development and evaluation of deterministic models for predicting the weights of fouling deposits from coal combustion: U.S. Geological Survey Open-File Report 89-208, 155 p.

Fishman, N.S., Turner, C.E., Peterson, Fred, Dubiel, R.F., Finkelman, R B., Tabet, D.E., and Quick, J.C., 1999, Analcime in Wasatch Plateau coal, Utah: Geological Society of America Annual Meeting Abstracts with Program, v. 31, p. A-386.

Goldstein, J.I., Newbury, D.E., Echlin, Patrick, Joy, D.C., Fiori, Charles, and Lishin, Eric, 1981, Scanning electron microscopy and X-ray microanalysis: New York, Plenum, 673 p.

Honea, F.I., Montogemery, G.G., and Jones, M.L., 1982, Recent research on ash fouling in combustion of low rank coals, in Kube, W.R., Sondreal, E.A., and White, D.M., compilers, Technology and use of lignites, volume 1, Eleventh Biennial Lignite Symposium, Proceedings: Grand Forks Energy Technology Center IC 82/1, p. 504-545.

Laubach, S.E., Marrett, R.A., Olson, J.E., and Scott, A.R., 1998, Characteristics and origins of coal cleat-A review: International Journal of Coal Geology, v. 35, p. 175-207.

Reid, W.T., 1981, Coal ash-Its effects on combustion systems, in Elliot, M.A., ed., Chemistry of coal utilization-Second supplementary volume: New York, John Wiley and Sons, p. 1389-1445.

Turner, C.E., Finkelman, R.B., Fishman, N.S., Dubiel, R.F., and Peterson, Fred, 1997, Evolution of coal quality-An integrated approach: Geological Society of America Annual Meeting, Abstracts with Program, p. A-260.

Vaninetti, G.E., and Busch, C.F., 1982, Mineral analysis of ash data-A utility perspective: Journal of Coal Quality, v. 1, p. 22-31.

Young, R.G., 1955, Sedimentary facies and intertonguing in the Upper Cretaceous of the Book Cliffs, Utah-Colorado: Geological Society of American Bulletin, v. 66, p. 177-202. 